\title{
Manual-scanning optical coherence tomography probe based on position tracking
}

\author{
Jian Ren, ${ }^{1, *}$ Jigang Wu, ${ }^{1}$ Emily J. McDowell, ${ }^{2}$ and Changhuei Yang ${ }^{1,2}$ \\ ${ }^{1}$ Department of Electrical Engineering, California Institute of Technology, 1200 East California Boulevard, \\ Pasadena, California 91125, USA \\ ${ }^{2}$ Department of Bioengineering, California Institute of Technology, 1200 East California Boulevard, \\ Pasadena, California 91125, USA \\ *Corresponding author: jren@caltech.edu
}

Received July 23, 2009; accepted August 29, 2009; posted October 1, 2009 (Doc. ID 114485); published October 29, 2009

\begin{abstract}
A method based on position tracking to reconstruct images for a manual-scanning optical coherence tomography $(\mathrm{OCT})$ probe is proposed and implemented. The method employs several feature points on a hand-held probe and a camera to track the device's pose. The continuous device poses tracking, and the collected OCT depth scans can then be combined to render OCT images. The tracking accuracy of the system was characterized to be about $6 \mu \mathrm{m}$ along two axes and $19 \mu \mathrm{m}$ along the third. A phantom target was used to validate the method. In addition, we report OCT images of a 54-stage Xenopus laevis tadpole acquired by manual scanning. (C) 2009 Optical Society of America

OCIS codes: $170.3880,170.4500,170.2150,150.6910,330.4150,100.4999$.
\end{abstract}

Optical coherence tomography's (OCT's) noninvasive nature and high resolution have made it an important modality in the field of biomedical imaging since the 1990s [1]. Aside from ophthalmology applications $[2,3]$, OCT has been implemented in various probes and endoscopic formats for many different applications $[4,5]$. Various methods for sweeping the OCT probe beam to accomplish scans have been developed over the years, most involving some form of mechanical actuation. While these systems allow for good scan control and render high-quality OCT images, they do come with the associated cost of additional hardware that must be integrated onto the probes. A hand-held OCT probe, which can be manually swept over the region of interest by the user to provide an OCT scan of the biological structures along its motion trajectory, could potentially simplify the image acquisition.

Here, we report what we believe to be a new OCT probe design based on position tracking to achieve the above manual-scanning capability. We developed an optical monitoring system that can continuously track the position and orientation (henceforth referred to as the pose) of a simple OCT probe while the probe is manually scanned over a region of interest. Both planar 2D images and volumetric 3D images could be reconstructed by orienting each OCT depth scan (A scan) according to their individual spatial location and direction as resolved from the poses of the hand-held device. Unlike mechanically actuated OCT probes that reconstruct images based on pre determined scan patterns [6-9], this method can objectively track and measure the actual scan pattern regardless of the scanning mechanism.

This design concept has three major advantages. First, the construction of such an OCT probe can be fairly, simple since the user will provide the actuation force; the low associated fabrication cost is advantageous for applications that call for disposable probes. Second, the absence of a mechanical actuation system allows for very lightweight OCT probe designs, granting the user greater control finesse during probe usage. Third, this design has significant flexibility as it allows the user to manually scan the region of his/her interest along almost any arbitrary pattern, as long as the trajectory of the device is within the tracking frame of the monitoring system.

The implemented tracking system is schematically illustrated in Fig. 1. Four IR LEDs centered at $950 \mathrm{~nm}$ were mounted on a tetrahedron frame to serve as feature points. Taking one of the LEDs as the origin of the reference frame, the coordinates for these feature points were $(0,0,0),(43 \mathrm{~mm}, 0,0)$, $(0,43 \mathrm{~mm}, 0)$, and $(0,0,43 \mathrm{~mm})$, respectively. An OCT needle probe was attached to this frame along the direction of $(-1,-1,-1)$. A monochrome complementary metal-oxide semiconductor (CMOS) camera with a focal length of $25.4 \mathrm{~mm}$ was placed about $51 \mathrm{~cm}$ away from the hand-held probe. Its frame rate was configured at $30 \mathrm{fps}$ with an array of 1280 $\times 1024$ pixels. The pixel size is $5.2 \mu \mathrm{m}$. With this optical arrangement, a plane $51 \mathrm{~cm}$ from the camera was recorded with a magnification factor of $\sim 0.05$

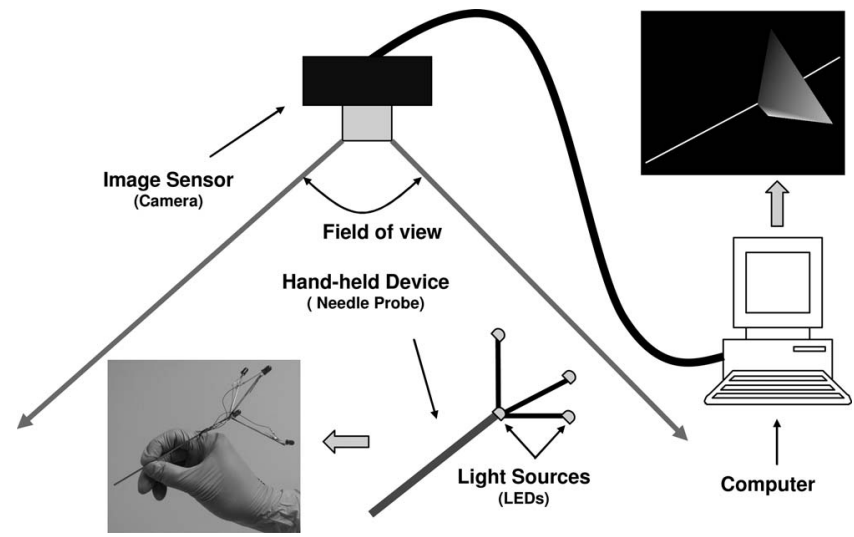

Fig. 1. Schematic of the tracking system. The left bottom inset is a photo of our prototype hand-held probe. The right top inset is a $3 \mathrm{D}$ rendering of the probe by the tracking system. 
and the achieved field of view was $13.3 \mathrm{~cm} \times 10.6 \mathrm{~cm}$. The camera captured 2D images of the feature points. The positions of these four feature points in the recorded images provide sufficient information for the determination of the probe's pose in three dimensions.

In the first stage of pose determination, we employed a pair of blob detection and tracking programs to locate the feature points, which appeared as bright circular areas (blobs), in each frame and track their correspondence among a sequence of frames. We then determined the location of each feature point with subpixel resolution $(\sim 0.03$ pixels $)$ by applying a centroid-based estimation algorithm in a surrounding square region after the center of the feature point had been approximately identified by the programs. Next, we employed a pose estimation algorithm known as pose from orthography and scaling with iterations (POSIT) [10] to compute the pose. The tracking system allowed us to track the probe's pose at intervals limited only by the camera's frame rate.

Our OCT probe consisted of a needle, $1.2 \mathrm{~mm}$ in diameter and $63.5 \mathrm{~mm}$ in length. A 3-mm-long, 0.29 pitch gradient-index lens connected to a single-mode fiber via a glass ferrule was housed within the needle. This probe focused light at a working distance of $3.9 \mathrm{~mm}$ (in air) ahead of its tip with a spot size of $19 \mu \mathrm{m}$. A swept laser centered at $1310 \mathrm{~nm}$ with a scan range of about $100 \mathrm{~nm}$ and an average power of $8.5 \mathrm{~mW}$ served as the OCT light source. The depth scan acquisition rate (A-scan rate) was $333 \mathrm{~Hz}$. The sensitivity of this OCT probe was measured to be $95 \mathrm{~dB}$. Concurrent with the pose tracking, our OCT probe continuously acquired A scans and transferred the data into the computer.

To examine the accuracy of our pose tracking system, we mounted the probe onto a mechanical stage that was translated over a length of $1 \mathrm{~mm}$ along the scan direction (orthogonal to the main axis of the probe) in increments of $10 \mu \mathrm{m}$. At each location, a group of 100 images was captured as a sufficient statistical ensemble and analyzed for pose estimation. For each sample image, we varied the size of the selected square regions around each identified feature point from $3 \times 3$ to $201 \times 201$ pixels during feature point centroid computations.

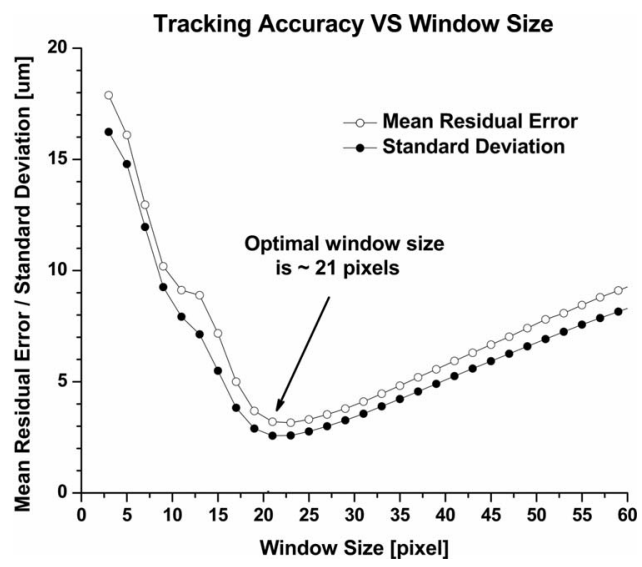

Fig. 2. Tracking accuracy characterization.
For each window size, we computed the mean residual error and the standard deviation of the estimated displacements. As the probe was translated in this experiment via a mechanical stage, its position was known at all times. We refer to the difference between this known position and the position computed by the tracking system as the residual error. The average of this error over the set of 100 images at each location is defined as the mean residual error. In effect, this quantity is a measure of the systematic positional error. The computed position varied from image frame to image frame as well, even when the probe was fixed in position. We refer to this variation as the standard deviation. This quantity is a measure of the random error associated with the measurement process. Figure 2 shows the behavior of these two error metrics (averaged over all the locations) as a function of the selected window size. We can see that both curves achieved minima at a window size of 21 pixels. This directed our window size choice for the pose estimation process. Here, we define the tracking accuracy as the sum of the above two types of errors. As indicated in Fig. 2, we were able to determine the position of the probe with an accuracy of $5.8 \mu \mathrm{m}$ at the optimal window size. We further evaluated our tracking ability perpendicular to the scan direction (i.e., along the OCT probe's main axis) via a similar experiment and determined the tracking accuracy along that axis to be $18.8 \mu \mathrm{m}$.

To demonstrate that this tracking approach can indeed be employed to generate reasonable OCT images, we performed a number of scans on a phantom sample. The phantom sample consisted of an agarose gel block embedded with $0.2 \mu \mathrm{m}$ latex microspheres

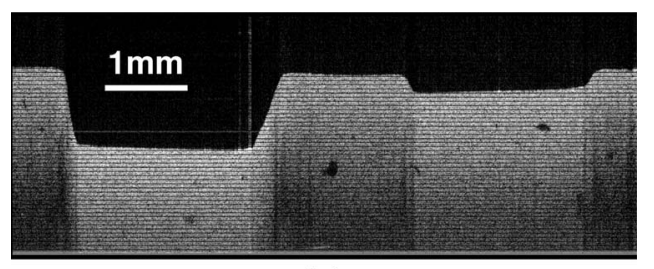

(a)

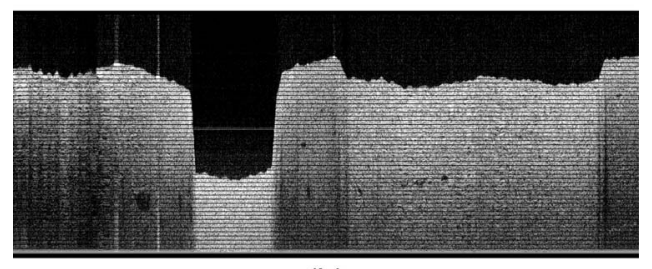

(b)

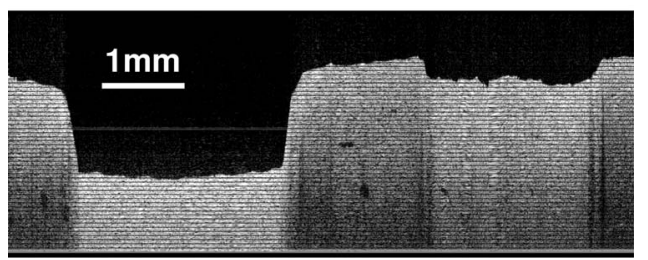

(c)

Fig. 3. (a) Target phantom image by a motorized stage scanning (control group). (b) Target phantom image by manual scanning and reconstructed based on time order. (c) Target phantom image by manual scanning and reconstructed based on estimated displacement. 


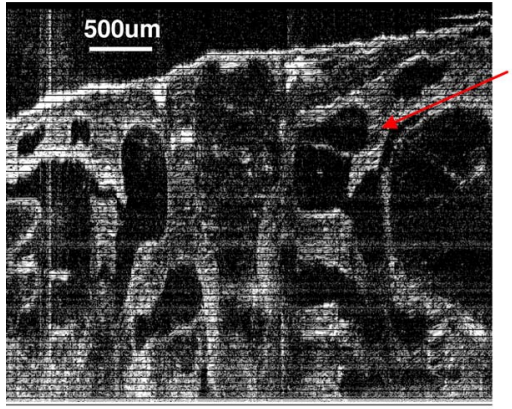

(a)

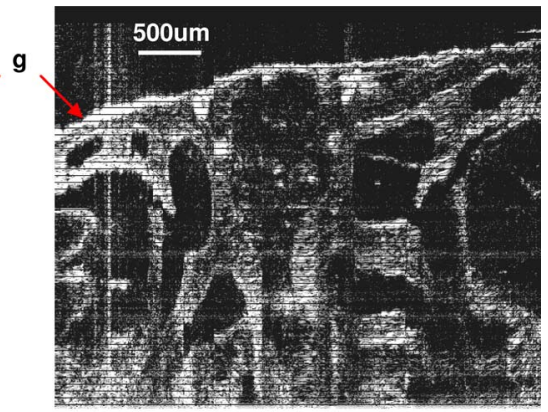

(b)

Fig. 4. (Color online) OCT images of a 54-stage Xenopus laevis tadpole specimen: (a) OCT image acquired by manually scanning without tracking correction and (b) OCT image acquired by manually scanning with tracking correction; g, gill pockets.

at a $0.66 \%$ volume concentration. Two grooves, roughly $2.5 \mathrm{~mm}$ wide, were carved on the surface and spaced $2.2 \mathrm{~mm}$ apart. The first groove was $1.3 \mathrm{~mm}$ deep, and the second was $0.3 \mathrm{~mm}$ deep. We first translated the probe across the surface of this sample via a motorized translation stage. The collected OCT image from this scan is shown in Fig. 3(a) and serves as the control. Next, we held the probe by hand and manually scanned the probe across the sample. This manual scan took $6 \mathrm{~s}$ and occurred over a length of $\sim 7.5 \mathrm{~mm}$. If we simply stack the collected A scans based on time order, the resulting OCT image [shown in Fig. 3(b)] corresponds poorly to our control image [Fig. 3(a)]. This is attributable to the fact that our manual scan motion exhibited significant velocity variations during the scan. In comparison, the construction of the OCT image based on accurate tracking of the probe's position rendered a significantly more accurate OCT image [Fig. 3(c)]. Hand scanning does not occur along strictly straight lines, and the scanning patterns we measured did have some deviation from the planes rendered here. To make the comparison more readable, we projected the $3 \mathrm{D}$ curved images to the same render plane as the control image, since the deviation was minor when compared to the scan range.

It is worth mentioning that the frame rate of the camera was relatively slow compared to the A-scan acquisition rate; thus, there were about ten $\mathrm{A}$ scans acquired between probe pose determination. We estimated the probe position for each A scan by linear interpolation from the known probe pose-time points. This interpolation might introduce some pose errors. The rendered image in Fig. 3(c) contains maximal possible interpolation-induced errors of $\sim 40 \mu \mathrm{m}$, which can be reduced with a camera of higher frame rate.

We next applied this method to image the gill pockets of a 54-stage Xenopus laevis tadpole. Figure 4(a) shows an uncorrected manual scan OCT image, and Fig. 4(b) shows a corrected version. The gill pocket on the upper left-hand side of each image appears to have a different shape between the two images, which is a result of this correction.

These experiments demonstrate that continuous pose tracking of an OCT probe is indeed useful as a means for accomplishing OCT image renderings. The position determination accuracy in our prototype is a sufficiently good match with the associated resolution of the OCT system. Our proposed system can be improved by incorporating a faster frame-rate pose tracker to allow for more accurate pose tracking. Pose accuracy can also be potentially improved by employing multiple cameras at different angles to monitor the probe's movement. This tracking system approach may be a good match for the confined work environment of the surgical suite, as the tracking camera could potentially be mounted on the suite's ceiling.

The authors thank Kevin Hsu at Micron Optics, Inc. for the loan of the swept source laser. We acknowledge discussions with Xiquan Cui and help from Shuyi Nie. This work was funded by the Biomimetic Micro-Electronic Systems Engineering Research Center (EEC-0310723) and a National Science Foundation (NSF) Career Award (BES-0547657).

\section{References}

1. D. Huang, E. A. Swanson, C. P. Lin, J. S. Schuman, W. G. Stinson, W. Chang, M. R. Hee, T. Flotte, K. Gregory, C. A. Puliafito, and J. G. Fujimoto, Science 254, 1178 (1991).

2. B. Povazay, K. Bizheva, B. Hermann, A. Unterhuber, H. Sattmann, A. F. Fercher, W. Drexler, C. Schubert, P. K. Ahnelt, M. Mei, R. Holzwarth, W. J. Wadsworth, J. C. Knight, and P. S. Russel, Opt. Express 11, 1980 (2003).

3. S. Han, M. V. Sarunic, J. Wu, M. Humayun, and C. H. Yang, J. Biomed. Opt. 13, 020505 (2008).

4. M. V. Sivak, K. Kobayashi, J. A. Izatt, A. M. Rollins, R. Ung-Runyawee, A. Chak, R. C. K. Wong, G. A. Isenberg, and J. Willis, Gastrointest. Endosc. 51, 474 (2000).

5. B. E. Bouma, G. J. Tearney, H. Yabushita, M. Shishkov, C. R. Kauffman, D. D. Gauthier, B. D. MacNeill, S. L. Houser, H. T. Aretz, E. F. Halpern, and I. K. Jang, Heart 89, 317 (2003).

6. G. J. Tearney, S. A. Boppart, B. E. Bouma, M. E. Brezinski, N. J. Weissman, J. F. Southern, and J. G. Fujimoto, Opt. Lett. 21, 543 (1996).

7. T. Q. Xie, H. K. Xie, G. K. Fedder, and Y. T. Pan, Appl. Opt. 42, 6422 (2003).

8. X. M. Liu, M. J. Cobb, Y. C. Chen, M. B. Kimmey, and X. D. Li, Opt. Lett. 29, 1763 (2004).

9. J. G. Wu, M. Conry, C. H. Gu, F. Wang, Z. Yaqoob, and C. H. Yang, Opt. Lett. 31, 1265 (2006).

10. D. F. Dementhon and L. S. Davis, Int. J. Comput. Vis. 15, 123 (1995). 